\title{
Erstnachweis von Anisodactylus signatus (Panzer, 1797) (Coleoptera: Carabidae) in Schleswig-Holstein
}

\author{
First record of Anisodactylus signatus (Panzer, 1797) \\ (Coleoptera: Carabidae) in Schleswig-Holstein
}

\begin{abstract}
Hannes Hoffmann ${ }^{(1)}$
Institut für Natur- und Ressourcenschutz, Abteilung für Landschaftsökologie, Christian-Albrechts-Universität zu Kiel, Olshausenstr. 75, 24118 Kiel, h.hoffmann@ecology.uni-kiel.de
\end{abstract}

\author{
Schlüsselwörter: \\ Autobahn, Blühfläche, \\ Klimawandel, Laufkäfer, \\ Rebhuhn Verschleppung, \\ Umweltmaßnahme
}

\begin{abstract}
Zusammenfassung
Anisodactylus signatus (Panzer, 1797) konnte erstmals für Schleswig-Holstein nachgewiesen werden. Das Weibchen wurde auf einer Blühfläche nördlich von Neumünster mit Bodenfallen gefangen. Es werden Angaben zum Fundort, Verbreitung und Habitatansprüchen geliefert.
\end{abstract}

\begin{abstract}
Anisodactylus signatus (Panzer, 1797) was discovered in the province of Schleswig-Holstein (Germany) for the first time. The female was caught on a sown wildflower field north of Neumünster using pitfall traps. Details of the locality, distribution and habitat requirements are given.
\end{abstract}

Am 30.5.2017 konnte vom Autor ein weibliches Individuum von Anisodactylus signatus (Panzer, 1797) in einer Bodenfalle auf einer Blühfläche nördlich von Neumünster nachgewiesen werden. Diese befindet sich etwa 700 m nordöstlich der Autobahnabfahrt, NeumünsterNord, und ist etwa einen halben Hektar groß. Im Zentrum der Blühfläche befindet sich ein Kleingewässer. Die Blühfläche wurde erstmalig im Sommer 2016 im Rahmen des vom Land Schleswig-Holstein geförderten Rebhuhnprojektes angelegt. Seitdem unterlag die Fläche keinerlei Nutzung, sodass sie im Sommer 2017 von einer fast einen Meter hohen und dichten Vegetation geprägt wurde (Abb. 1 \& 2). Dominierende Pflanzenarten waren Daucus carota, Stellaria graminea, Trifolium repens, Trifolium pratense, Lotus corniculatus, Plantago lanceolata, Agrostis gigantea, Alopecurus pratensis und Rumex crispus. Umgeben war die Blühfläche von Wintergerste. Im Jahr 2018 wird die Blühfläche umgebrochen, neu eingesät und anschließend erneut mit Bodenfallen beprobt. Anisodactylus signatus ist eine typische Art landwirtschaftlich geprägter Gebiete auf sandig-lehmigen Bö- den, wobei auch weitere sporadische Vorkommen in weiteren Lebensräumen der offenen Kulturlandschaft bekannt sind (Baehr 1980, Fritze 2017). Nach Fritze (2017) sind ausgedehnte Saumstrukturen oder Brachen als besonders bedeutend für die Art einzustufen und sollten zu deren Schutz gefördert werden. Weiterhin gibt es auch Nachweise an Küsten und Ufern von Seen und Tümpeln (Turin 2000, GAC 2009).

Anisodactylus signatus ist eine transpaläarktisch verbreitete Art, die von der Iberischen Halbinsel bis nach Japan nachgewiesen ist (Wrase 2004, Lorenz 2015). In Europa fehlt die Art weitestgehend im Norden (in Dänemark nur zwei Funde um 1860), Nordwesten und im Süden (Turin 2000, Fritze 2017). In Deutschland liegt ihr Verbreitungsschwerpunkt im Südwesten (Fritze 2017). Aus den nördlichen und westlichen Bundesländern liegen entweder keine oder nur sehr alte Funde vor (Abb. 3). In Schleswig-Holstein wurde die Art von Lohse gestrichen, da die Existenz des einzigen Fundes („Hamburg, 1 Exemplar, ex Coll. Gebien in Sammlung Frey“) Herrn Gebien völlig unbekannt war und dort sicher nicht 


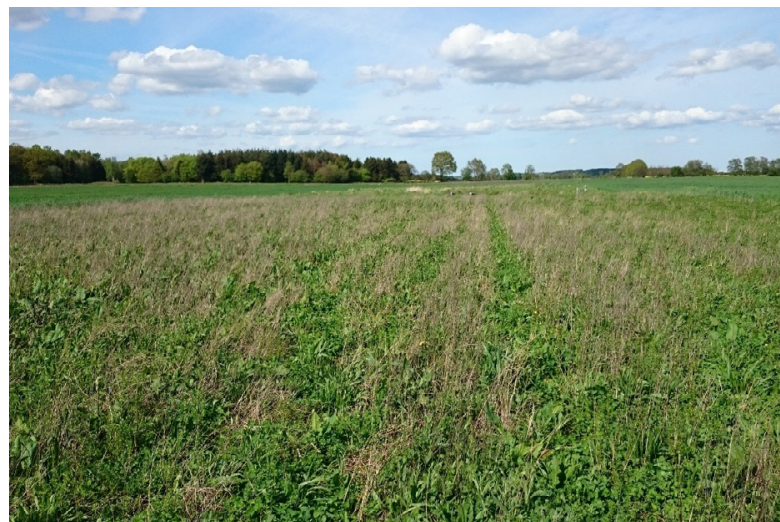

Abb. 1: Fundort von Anisodactylus signatus (Panzer, 1797) nördlich von Neumünster zum Zeitpunkt des Aufstellens der Bodenfallen am 15.05.2017

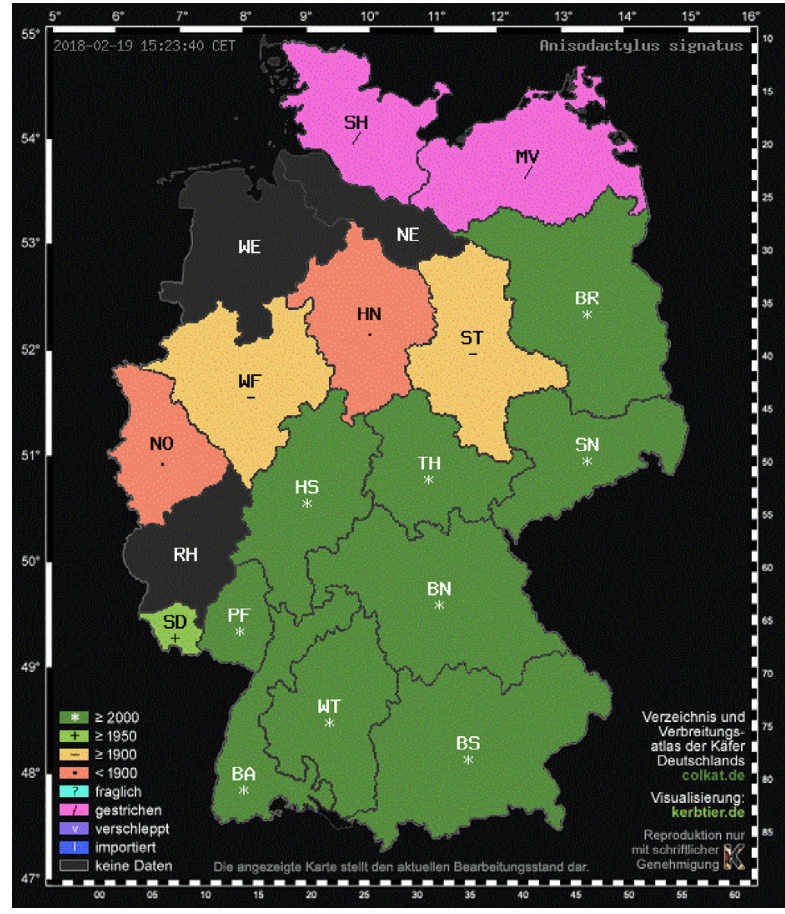

Abb. 3: Aktuelle Verbreitung (Katalogstatus) von Anisodactylus signatus (Panzer, 1797) in Deutschland (Benisch 2018, Bleich et al. 2018).

gefunden wurde (Horion 1941, Lohse 1954). Demnach gilt der vorliegende Fund als Erstnachweis der Art für Schleswig-Holstein. Die aktuellsten und am nächstgelegensten Nachweise von A. signatus befinden sich in Hessen, Thüringen, Sachsen und in Brandenburg an der Elbe und Oder (Esser 2005, Bleich et al. 2018).

Prinzipiell erscheinen die Ansprüche von A. signatus (im Süden eher auf sandigen Äckern, im Norden in nassen Lebensräumen) widersprüchlich. Der aktuelle Nachweis auf der Blühfläche, die um ein Kleingewässer angelegt wurde, bietet beide potenziellen Lebensräume

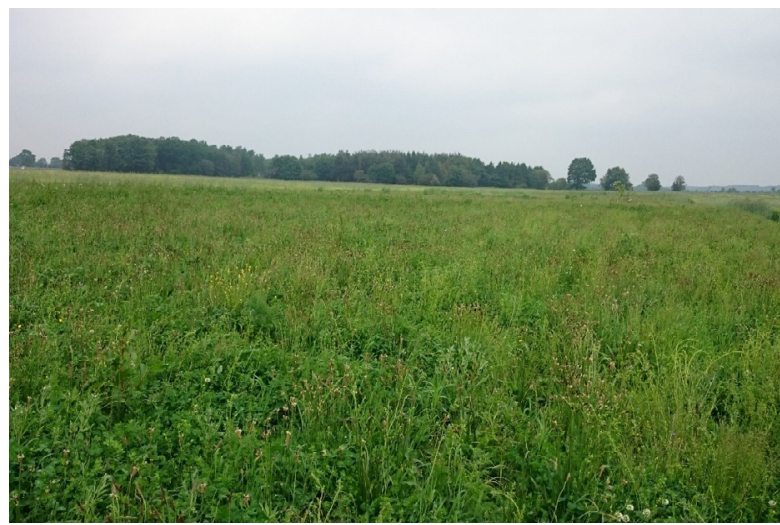

Abb. 2: Fundort von Anisodactylus signatus (Panzer, 1797) nördlich von Neumünster zum Zeitpunkt der ersten Leerung der Bodenfallen am 30.05.2017.

auf engstem Raum. Diese unterschiedlichen Bedingungen zeigen auch weitere Nachweise von Harpalus rufipes (De Geer, 1774), Chlaenius nigricornis (Fabricius, 1787) und Anisodactylus binotatus (Fabricius, 1787) auf dem gleichen Standort. Der aktuelle Nachweis aus Schleswig-Holstein ist auch dahingehend schwierig $\mathrm{zu}$ interpretieren. Generell ist eine Ausbreitung wie aktuell in Brandenburg nicht auszuschließen, aber aufgrund der großen Distanz zu weiteren Vorkommen und des Fundortes an der Autobahn wird, vorbehaltlich der weiteren Entwicklung, von einer Verschleppung ausgegangen.

Das gefundene Individuum verbleibt in der Sammlung des Autors.

\section{Dank}

Mein herzlichster Dank geht an Tim Diekötter und Stephan Gürlich für die Durchsicht des Manuskriptes. Das Projekt wird gefördert durch das Ministerium für Energiewende, Landwirtschaft, Umwelt, Natur und Digitalisierung des Landes Schleswig-Holstein.

\section{Autor}

\section{Hannes Hoffmann ${ }^{\mathbb{1}}$}

Hannes Hoffmann ist Entomologe und beschäftigt sich hauptsächlich mit der Ökofaunistik der Kurzflügelkäfer (Staphylinidae) in Norddeutschland. Wissenschaftliche Untersuchungen führte er zum Einfluss von Agrarumweltmaßnahmen auf bodenbewohnende Arthropoden und von Renaturierungs- 
maßnahmen von Fließgewässern auf Lauf- und Kurzflügelkäfer durch. Weiteres Interesse besteht in der Erfassung und Förderung der Arthropodendiversität in Hamburg.

\section{Literatur}

Baehr M (1980) Die Carabidae des Schönbuchs bei Tübingen (Insecta, Coleoptera). 1. Faunistische Bestandsaufnahme. Beitrag zur Faunistik der Carabiden Württembergs 2. Veröffentlichungen für Naturschutz und Landschaftspflege in Baden-Württemberg 51/52: 515-600.

Benisch C (2018) Kerbtier.de: Käferfauna Deutschlands, www.kerbtier.de, letzter Zugriff am 14.10.2021.

Bleich O, Gürlich S, Köhler F (Stand: 19.2.2018) Entomofauna Germanica Online: Verzeichnis der Käfer Deutschlands, www.coleokat.de, letzter Zugriff am 14.10.2021.

Esser J (2005) Anisodactylus signatus (Panzer, 1796) (Col., Carabidae) ein Fund in Sachsen. Entomologische Nachrichten und Berichte 49:46.

Fritze M-A (2017) Tribus Anisodactylini. In: Trautner J (Hg.) Die Laufkäfer Baden-Württembergs. Band 1. Verlag Eugen Ulmer, Stuttgart, S. 453-457.

Gesellschaft für Angewandte Carabidologie (2009) Lebensraumpräferenzen der Laufkäfer Deutschlands: wissensbasierter Katalog. GAC, Filderstadt.

Horion A (1941) Faunistik der deutschen Käfer. Band 1: Adephaga - Caraboidea. H. Goecke, Krefeld.

Lohse GA (1954) Die Laufkäfer des Niederelbegebietes und SchleswigHolsteins. Verhandlungen des Vereins für naturwissenschaftliche Heimatforschung zu Hamburg 31: 1-39.

Lorenz W (2015) CarabCat: Global database of ground beetles (version Oct 2017). Species 2000 \& ITIS Catalogue of Life, www.catalogueoflife org, letzter Zugriff am 14.10.2021.

Turin H (2000) De Nederlandse loopkevers, verspreiding en oecologie (Coleoptera: Carabidae). KNNV, Utrecht.

Wrase D (2004) 20. Tribus: Harpalini. In: Müller-Motzfeldt G (Hg.) Die Käfer Mitteleuropas. Band 2: Adephaga-1. Carabidae (Laufkäfer), 2. Auflage. Spektrum, Akad. Verl., Heidelberg, S. 344-420.

\section{Open Access}

> Der Artikel ist unter der Creative-Commons-Lizenz Namensnennung 4.0 International veröffentlicht. Den Vertragstext finden Sie unter: https://creativecommons.org/licenses/by/4.0/deed.de. Bitte beachten Sie, dass einzelne, entsprechend gekennzeichnete Teile des Artikels von der genannten Lizenz ausgenommen sein bzw. anderen urheberrechtlichen Bedingungen unterliegen können. 